\title{
Research on Mining and Application of Group Events Based on Network Public Opinion Big Data
}

\author{
Weimin $\mathrm{Gao}^{1,2}$, Jiaming Zhong ${ }^{3, *}$ and Yuan $\mathrm{Xiao}^{3}$ \\ ${ }^{1}$ School of Computer Science and Engineering, Central South University, \\ ChangSha, China 410083 \\ ${ }^{2}$ Department of Computer Science and Engineering, Hunan Institute of Technology, \\ Heng Yang, China 421002 \\ ${ }^{3}$ College of Economic and Management, Xiangnan University, Chenzhou, China \\ 423000 \\ E-mail:gwm@hnit.edu.cn; jmzhongcn@163.com; yuanyuan_8603@qq.com \\ ${ }^{*}$ Corresponding Author
}

Received 26 May 2021; Accepted 22 June 2021;

Publication 14 October 2021

\begin{abstract}
Network Public Opinion is significant in maintaining social harmony and stability and promoting transparency in government affairs. However, with the development of economy and transformation of society, our country has entered a high-risk period, which is full of unexpected public events. Unexpected mass accidents also cause hot discussions among the Internet users once they are exposed on the network. Different ideas, opinions, emotions, and attitudes about unexpected public events will be collected and collide on the Internet. It makes Network Public Opinion play an increasingly important role in the evolution of unexpected public events. It could promote the spread and upgrade of unexpected public events and bring more and more profound influence on to our social life. We use the case study method to analyze and solve the problems by applying the dynamic principles of the
\end{abstract}

Journal of Web Engineering, Vol. 20_6, 1885-1908.

doi: 10.13052/jwe1540-9589.2069

(C) 2021 River Publishers 
SIR epidemic model, comprehensively considering the social environment and various influencing factors, and constructing a mathematical model for the spread of network group events. The study uses Matlab to simulate the change trajectory of the number of participants in the network group events. By adjusting the number of contacts $\varphi$ in the model, the development of network group emergencies can be effectively controlled and managed. As long as the government takes timely intervention measures, the dissemination of network group events can be basically controlled. Combined with public opinion big data to discover the important factors affecting the spread of public opinion, the control effect is obvious..

Keywords: Mining and application, network group events, simulation, SIR (Susceptible Infected Recovered Model).

\section{Introduction}

The frequent occurrence of various mass incidents in recent years has caused heavy losses to people's lives and property as well as to economic and social development. With a rapid increase in the number of netizens in my country and the popularization of mobile smart devices, new media tools such as social networks have gradually penetrated into people's lives. Mass incidents are spreading faster online, and people's attention to mass incidents is increasing. If people's governments at all levels do not actively respond to and effectively adapt to the impact of Network Public Opinion, and do not establish a feasible Network Public Opinion early warning mechanism for mass incidents as soon as possible, then the potential social public opinion will become the catalyst and fuse of the emergence of sudden mass incidents.

Research of the domestic academic circles on group events mainly focuses on three aspects. Firstly is the root cause of mass incidents [1]; secondly is the classification of mass incidents [2]; and thirdly is the handling of mass incidents [3]. However, with the advent of the internet age, network mass incidents have gradually become an important research direction in studying group events as a new problem in social development. The theoretical basis mainly focuses on crisis life cycle theory, complex system theory, and silent spiral theory. Among them, the research of Pengbo [4] is more comprehensive. He analyzed (a) the causes of online mass incidents, and (b) the internal laws of online mass incidents, and proposed preventive strategies. Peng Zhihui [5] pointed out that Network Public Opinion has a huge impact on the development and evolution of mass incidents. Network Public Opinion 
often leads mass incidents to a vicious development direction, and sometimes even directly triggers mass incidents. ShiBo [6] analyzed the internal logic of the evolution of Network Public Opinion in public crisis events; made an indepth analysis of the emergence, evolution, and reasons of Network Public Opinion in public crisis events; and proposed four integrated management operation mechanisms - early warning mechanism, disposal mechanism, aftermath mechanism, and an integrated coping mechanism framework. Yang Jiuhua [7] pointed out that the online launching mode of stakeholder groups, the Network Public Opinion triggering mode, the vicious development mode of online rumors promoting the vicious development of mass incidents, and the mode of hostile forces initiating mass incidents through the Internet are the four modes of online mass incidents. He Guoping [8] believes that the "mobilization mode" that promotes the generation of online mass incidents includes four mobilization modes: induced mobilization, focused mobilization, public relations mobilization, and anger-venting mobilization. Harvard University psychology professor Stanley Milgram [9] discovered the phenomenon of "six degrees of separation" (also known as the "small world" phenomenon).

In this paper, we focuses on the current situation of frequent online public opinion crises, and deeply digs into the key factors of online public opinion triggering mass incidents and their connections. Additionally, it aims to prepare conditions and propose countermeasures to reasonably control the evolution and escalation of public opinion and prevent mass incidents.

This study uses the case study method to solve the problems. The application of the SIR epidemic model is helpful to comprehensively consider the social environment and various influencing factors, and construct a mathematical model for the spread of network group events. Using Matlab to simulate the change trajectory of the number of participants in network group events, by adjusting the number of contacts $\varphi$ in the model, the development of network group emergencies can be effectively controlled and managed. As long as the government takes timely intervention measures, the dissemination of network group events can be basically controlled. Combined with public opinion big data to unearth important factors that affect the dissemination of public opinion, the control effect is obvious.

In summary, our major contributions are:

- Analysis of the characteristic information elements of group events that were excavated from the big data of online public opinion, and establishing a dynamic network public opinion monitoring index system through the characteristic factors of the evolution and development of online public opinion. 
- Proposing a SIR-based Network Group Event propagation model, which can well simulate the spread of sudden network mass incidents. We have established public opinion big data, defined adjustment parameters through hot data mining, and fitted the simulation results with the actual propagating trend of public opinion events, which proved the effectiveness of the model.

- Demonstrating, by the use of Matlab simulations, that the SIR-based Network Group Event propagation model performs remarkably better than Support-Neutral-Oppose (SNO) under realistic PX network group events. Especially, SIR is about $10 \%$ faster than SNO in convergence speed.

The remainder of this study is structured as follows. In Section 2, we describe our design motivation. The design detail of the mining system and model is presented in Sections 3, 4, and 5. In Section 6, we show the experimental results of Matlab simulation. Finally, we conclude the study in Section 7.

\section{Background and Motivation}

In this section, we show the current status of the application of big data in Network Public Opinion, the main problems that exist, and the motivation of research.

\subsection{The Present Situation of the Application of Big Data in Network Public Opinion}

The application of big data is divided into two parts: big data technology and big data thinking. The two merge and function together to form the application of big data, which has a huge impact on many fields including the Network Public Opinion research. The process of the Network Public Opinion research can be roughly divided into Network Public Opinion monitoring, Network Public Opinion analysis, and Network Public Opinion early warning.

Network Public Opinion monitoring is the monitoring of the frequency and trend of network hot public opinion within a certain period, emphasizing the discovery of new information and paying attention to specific hotspots. The main point is the collection and storage of Network Public Opinion information. Big data Network Public Opinion monitoring mainly uses targeted site information capture, supplemented by comprehensive real-time 
monitoring; aggregated content social networking site information collection and summary collection; and other technologies, combined with traditional manual monitoring, to achieve capture efficiency and coverage. The research mainly focuses on three aspects: Network Public Opinion monitoring system, Network Public Opinion monitoring model, and Network Public Opinion monitoring algorithm [10].

Network Public Opinion analysis is to analyze the topics, events, and individuals that play a key role in Network Public Opinion monitoring. Factors such as groups are used as analysis objects, and on the basis of data analysis, the theoretical methods of various disciplines are used to judge the situation and trend of Network Public Opinion [11]. In terms of online public opinion analysis, the main technical means of big data analysis is data mining, which reveals the hidden process of information aggregation and refinement. At present, the most mature big data technology applied in Network Public Opinion analysis is the Hadoop solution.

Network Public Opinion early warning is usually based on Network Public Opinion analysis; that is, the level of online public opinion information is evaluated according to the degree of attention, transmission speed, and the degree of influence of the public opinion information in the Network Public Opinion event, and corresponding early warning thresholds are set. Big data provides technical support for Network Public Opinion warning and promotes the reform and innovation of Network Public Opinion warning. Looking at the relevant research of big data in the early warning of Network Public Opinion, it mainly discusses the two issues of Network Public Opinion early warning model and Network Public Opinion early mechanism.

\subsection{Main Problems and Motivations}

The main problems are:

(i) Network Public Opinion big data monitoring technology needs to be updated. At present, big data technology is in terms of online public opinion monitoring, and it has not been able to achieve optimal compatibility and efficiency, and at the same time, it has not been able to achieve compatibility between maximizing information collection and protecting privacy.

(ii) Network Public Opinion big data analysis technology needs to be improved. At present, big data of online public opinion includes text, video, image, audio, etc., while the existing big data analysis technology of online public opinion mostly uses text classification methods for data 
mining, which cannot perform good analysis on data other than text. In addition, in the face of massive high-speed and complex network public opinion data that needs to be processed and analyzed in real time, there is nothing that can be done.

Based on the aforementioned analysis, we draw the following conclusions: (i) the proposed model can well simulate the spread of sudden network mass incidents, and (ii) it can propose characteristic information that has a significant impact on the spread of public opinion from the big data of online public opinion. These conclusions motivate us to design a novel model and group event mining system that can control the spread of public opinion on network group events.

\section{Characteristic Factors of Evolution of Network Public Opinion, and Dynamic Monitoring Index System}

Network Public Opinion means that people can use the internet to publish relevant speeches, opinions, etc., and express various emotions on a matter anytime and anywhere; and gather, influence, and drive a large number of netizens with the same views in a short period to form a network force. The connotation and scope of Network Public Opinion are very broad, including Network Public Opinion events, online mass events, new media events, etc. At present, government websites, blogs, Weibo, WeChat, forums, post bar, QQ, and live webcast platforms have become the main ways for people to express public opinions and wishes [12].

From the perspective of open system analysis of Network Public Opinion, Network Public Opinion has obvious group characteristics. The main characteristics of its evolution are summarized as autonomy and controlling, interaction and suddenness, concealment and explicitness, irrationality and emotion diversification, diversity and diversification, and expansion of public sentiment momentum [13]. Due to the aforementioned characteristics of Network Public Opinion, there is a lack of good and bad news in Network Public Opinion, and there is a flood of emotional and irrational information. Therefore, in the face of the complex Network Public Opinion, the government needs to understand public opinions behind the Network Public Opinion and explore new countermeasures.

The four major elements of the evolution of Network Public Opinion are: events, netizens, media, and content. Network Public Opinion events have their life cycles, and they will all go from budding to disappearing, and their 


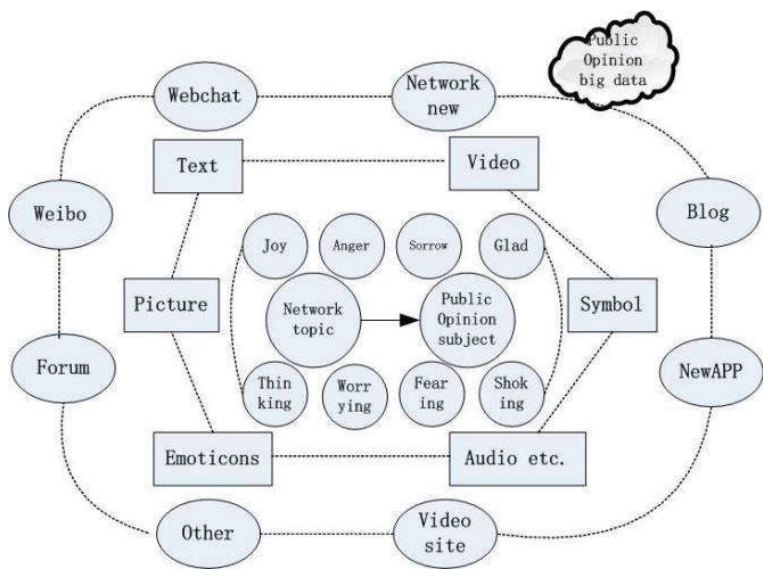

Figure 1 Elements of big data of Network Public Opinion.

development and evolution can be divided into several stages [14, 15]. The dynamic Network Public Opinion monitoring index system is divided mainly into:

- An indicator system based on social early warning research

- An index system based on subject classification

- An indicator system based on different entities of public opinion

- A Network Public Opinion monitoring index system based on the internal mechanism of Network Public Opinion.

Figure 1 shows the elements of big data of Network Public Opinion.

According to the life cycle theory, the development of everything has to go through the four stages of emergence, development, climax, and end. The evolution trend of Network Public Opinion in group events will also follow this theory. As we all know, stage characteristics are one of the most important characteristics of Network Public Opinion of mass events. It is divided into four stages: incubation period, outbreak period, development period, and fallback period. Different development stages present different characteristics, laws, and contents.

\section{Group Event Mining System of Network Public Opinion}

Mass incidents of Network Public Opinion refers to the fact that people use the Internet as a platform and use online forums, blogs, e-mails, online newsgroups, and other online tools to publish information about the upcoming or 
existing mass incidents and express their social and political attitudes [16]. The prediction system is the basis for the construction of the Network Public Opinion early warning mechanism for mass incidents [17]. It uses artificial intelligence and machine learning algorithms to automatically predict the masses. Early warning theory includes the following five stages: information judgment and analysis, information collection, formulation of relevant countermeasures, information transmission, and information feedback. During the operation of the entire early warning mechanism, correct judgment and analysis are made through information collection, and then information is transmitted, and finally the emergency measures are initiated.

According to the characteristics of the Network Public Opinion data and the final demand of the practical application of public crisis management, this study constructs a Network Public Opinion mining system, which is composed of data acquisition layer, data prepossessing layer, data storage layer, data intelligent analysis layer, public opinion information presentation layer, and application layer, as shown in Figure $2[18,19]$.

The Network Public Opinion information collection layer is the premise and guarantee of the Network Public Opinion monitoring system. It grasps the most comprehensive public opinion information at the collection stage. Since the distribution of Network Public Opinion information is relatively scattered, methods such as crawler scheduling, automatic crawling, automatic classification clustering, topic detection, and topic focusing can be used to obtain the basic data. The task of the data prepossessing layer is to filter and analyze the basic data, and use different data extraction methods to clean the metadata to ensure the quality and clustering effect of public opinion data [20]. The data storage layer implements data conversion and index management services. The main task of intelligent analysis of public opinion data is to use intelligent analysis algorithms to complete topic identification and tracking, hot topic discovery, and text tendency analysis. The public opinion information presentation layer is the part that displays the effect of the public opinion hot spot mining system and interacts with users. Functions of the Network Public Opinion mining system are as follows: emergency public opinion warning, hot topic Weibo and WeChat video presentation, statistical charts, and viewing [12].

From the modeling of Literature [21] and the Network Public Opinion simulation of mass event dissemination, as shown in Figure 3, only the public opinion spread from day 1 to day 13 is collected. The observation period of public opinion is short, and finally shows the trend of public opinion heat at a fixed value rather than tending to zero. Under the joint influence 


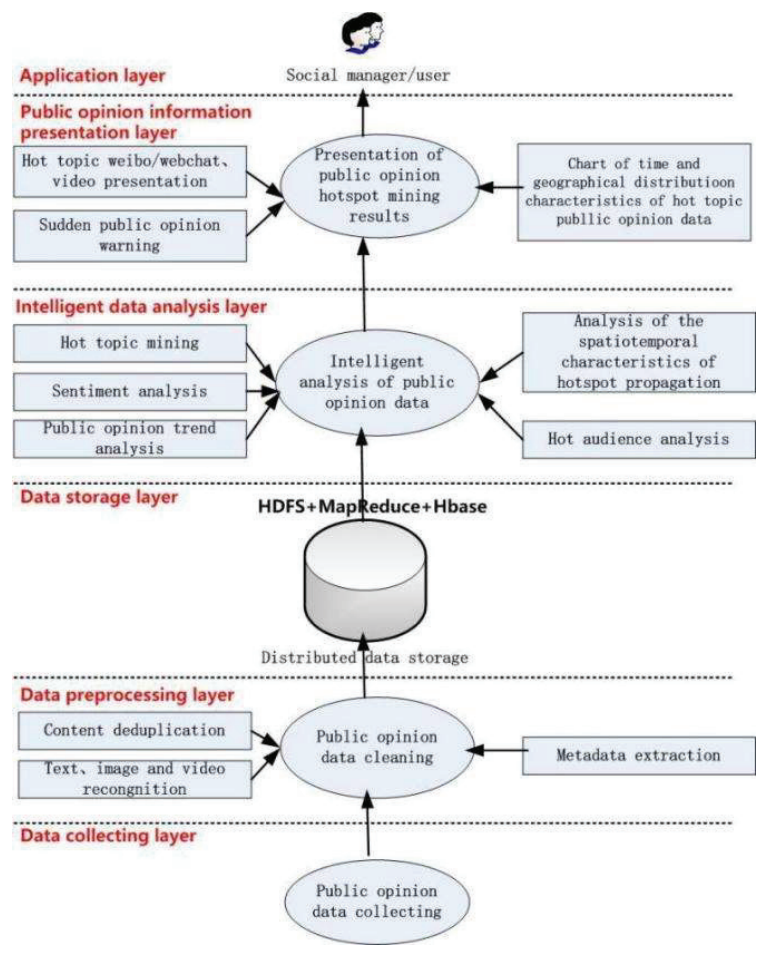

Figure 2 Network Public Opinion Group event mining system.

of events, netizens, media, the government, and the ecological environment of Network Public Opinion, the enthusiasm of public opinion on public emergencies quickly rises and reaches a peak, and then slowly decreases until it stabilizes at a low level. The changing trend of the spread curve of Network Public Opinion is consistent with the life cycle of public opinion development. In the aging period of public opinion, only a few event-related parties disseminate information, and public opinion is relatively low. Then, as the information of the event spreads, a large number of netizens who are onlookers become participants. Public opinion quickly enters the development and outbreak period. Finally, due to the resolution of the incident or the emergence of other new problems, netizens lose their enthusiasm for participating in the incident, and public opinion enters a period of decline and disappearance. Combining the comprehensive Network Public Opinion group event mining system mentioned above, through literature retrieval and simulation comparative analysis, it can be concluded that the three indicators of event sensitivity, government recognition, and Network Public Opinion 


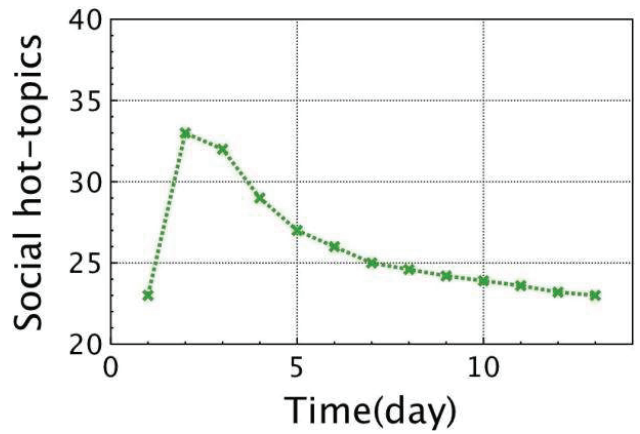

Figure 3 Development trend of Network Public Opinion heat of mass events.

ecological environment index have a greater impact on the spread of Network Public Opinion.

The sensitivity of mass incidents is determined by the attributes of the incident itself. It is the public perception of the sensitivity of the incident. Depending on the sensitivity of the incident, netizens, media, and the government will react differently to sudden group incidents $[22,23]$. When an incident involves some sensitive topics related to the conflict of interest or public safety, it is often easier to attract the attention of netizens, media, and the government; and the possibility of rising public opinion on the Internet is greater [13].

Government recognition is the degree of trust and recognition of social members of the government, and it is determined by the evaluation of netizens, opinion leaders, and the media's ability to handle government emergencies. The level of government recognition can have a significant impact on the guidance of Network Public Opinion.

The ecology of Network Public Opinion is a dynamic process, all through from the emergence of public opinion topics to attract the attention of netizens, the formation of public opinion, the upgrading of public opinion, to its decline. It affects all stages of the development of Network Public Opinion.

\section{Construction of the Dynamic Evolution Model of Network Public Opinion}

Traditional Network Public Opinion propagation models generally apply the "average field" assumption in system dynamics, which ignores the specific details between individual network nodes and assumes that nodes have a consistent average infection probability and immunity (or cure rate). Then 
it is concluded that the overall effect of node interaction in the network is equivalent to an "average field."

As the main participants in the dynamic evolution of online public opinion, the behavioral characteristics of individual citizens not only affect netizens' opinions, emotions, and expression of opinions, but also the interaction between netizens and the aggregation and integration of netizens' opinions. Ultimately, they also affect the network and the evolution trend of public opinion. The attention of online media, the continuity of reports, the openness of comments, etc., especially the tendency of media reports, affect the dynamic evolution of Network Public Opinion.

\subsection{Weisbuch-Deffuant Model}

The Weisbuch-Deffuant model [24] proposed by Weisbuch and Deffuant et al. is a typical social dynamics research paradigm used to describe the aggregation process of group opinions based on individual interaction. The assumptions of the model are as follows:

(1) The opinion held by the individual is a random number distributed between $[0,1]$

(2) Random interaction between individuals

(3) Two random individuals employ the following interaction rule: when the difference between the opinions of two individuals is less than a certain threshold w, they can realize the interaction of opinions; otherwise they will not interact.

\subsection{SNO Model}

Literature [25] proposed a dynamic evolution model of SNO Network Public Opinion based on complex network. The construction method is as follows:

(1) Carry out Network Public Opinion data collection, analysis, and processing, and then initialize the system control parameters.

(2) Select the (Newman \&Watts)NW small-world network model as the complex network structure model to analyze the network structure relationship.

(3) With reference to the SIR virus spreading model paradigm, the government, media, and netizens can choose to support neutral or opposing attitudes toward Network Public Opinion; set the government, media, and netizen participants in the subject's behavior attributes and environmental variables. 
(4) According to the interaction rules of the Weisbuch-Deffuant model, using fuzzy mathematics methods, assuming that the parameters obey the triangular fuzzy number or the trapezoidal fuzzy number, an improved rule for the interaction of the subject's opinion based on the Weisbuch-Deffuant model in the dynamic evolution process of Network Public Opinion is proposed.

(5) Set the parameters of each simulation model, start the simulation, and continuously adjust and optimize.

\subsection{SIR-based Network Group Event Propagation Model}

In 1927, Kermack and McKendrick [26] developed the SIR Cell Model when they were studying the black death epidemic in London, in which the most infectious diseases, such as smallpox, influenza, and hepatitis are cured with high immunity; therefore, the people who are cured are not healthy (susceptible) or sick (infective), and they are out of the infection system. The population within the epidemic scope of infectious diseases can be divided into three categories: susceptible, infective, and immune (or infection removed).

After mass emergencies occur, relevant public opinion information will spread quickly and negative public opinion information will intensify the development and escalation of mass emergencies, increase the difficulty of handling, and expand the scope of harm. The impact of the spread of Network Public Opinion on mass emergencies is quite similar to the spread of epidemic viruses. Participants in mass incidents are like sick people. They will affect the normal people through the spread of public opinion, allowing normal people to participate in the incident $[27,28]$. Based on the virus infection model, it establishes a model of the influence of public opinion spread on mass emergencies.

Model assumptions:

(1) The total number of people is N; after a mass event occurs, the population is divided into group event participants; that is, they have been infected by public opinion and can spread public opinion outside, and their proportion in the social group at time $t$ is $I(t)$.

(2) For non-participants, namely healthy people, the proportion in the social group at time $t$ is $R(t)$.

(3) For each participant, the average number of non-participants involved in mass events every day through the spread of Network Public Opinion is a constant $\mathrm{k}$, which is defined as the daily infection rate in this study. 
(4) Participants may use communication and persuasion every day. The proportion of withdrawal from group events to the total number of participants is $\alpha$. Since participants withdraw from group events and become non-participants, obviously $\frac{1}{\alpha}$ is the average influence period for the influence of this kind of public opinion spread on mass incidents.

According to the aforementioned assumptions, each participant in the group event can make $k \times R(t)$ non-participants of the group participate in the group event through Network Public Opinion dissemination every day. Because of the number of patients $N \times I(t)$, there is a total of there is a total of $\mathrm{k} \times \mathrm{N} \times \mathrm{I}(\mathrm{t}) . \mathrm{R}(\mathrm{t})$ per day. A bystander participates in a mass event, and the number of people who withdraw at the same time is $\alpha \times \mathrm{N} \times \mathrm{I}(\mathrm{t})$. $\mathrm{R}(\mathrm{t})$; therefore, $\mathrm{kNI}(\mathrm{t}) \mathrm{R}(\mathrm{t})-\alpha \mathrm{kNI}(\mathrm{t}) \mathrm{I}(\mathrm{t})$ is the increase in the rate of the participant's $\mathrm{N}_{\mathrm{i}}(\mathrm{t}) \mathrm{Ni}(\mathrm{t})$, namely:

$$
N \times \frac{d i}{d t}=k \times N \times R_{i}-\alpha \times N_{i}
$$

Also because

$$
I(t)+R(t)=1
$$

Suppose the proportion of participants at the initial moment $(\mathrm{t}=0)$ is $\mathrm{x} 0$, then

$$
\left\{\begin{array}{l}
\frac{d i}{d t}=k \times i \times(1-i)-\alpha \times i \\
x(0)=x_{0}
\end{array}\right.
$$

Solving this equation, we can get the influence model of Network Public Opinion spreading on mass emergencies:

$$
I(t)= \begin{cases}{\left[\frac{k}{k-\alpha}+\left(\frac{1}{x_{0}}-\frac{k}{k-\alpha}\right) \times e^{-(k-\alpha) t}\right]^{-1},} & k \neq \alpha \\ x(0)=x_{0}, & k=\alpha\end{cases}
$$

Let $\phi=\frac{k}{\alpha}$ be the average number of effective contacts of participants in each event during an infectious period, called the number of contacts. It can be derived from Equations (3) and (4),

when $t \rightarrow \infty$

$$
\mathrm{I}(\infty)= \begin{cases}1-\frac{1}{\emptyset} & \emptyset \geq 1 \\ 0 & \emptyset \leq 1\end{cases}
$$


This shows that the number of contacts $\varphi=1$ is a threshold. The increase or decrease of $\mathrm{I}(\mathrm{t})$ depends on the size of $\mathrm{x}_{0}$, that is, whether the event can be subdued depends on the size of the group event.

The sensitivity of the event is equivalent to the degree of Network Public Opinion attention [26]. The model construction method depends on the media, that is, on the weight of traditional news media and online news media used by participants in the process of public opinion information dissemination. According to the SIR infectious disease model, assuming ES represents the Event sensitivity, ES obeys normal distribution, and ES can be expressed as

$$
E(E S)=N \times \varphi
$$

The SIR model assumes that the average duration of infection spreaders is $\mathrm{d}$, which is a constant. $\mathrm{d}$ represents the average infection time of the infection spreaders. Recovering to the immune population is a first-order negative feedback process. Assuming GR represents the government's approval rate, then GR can be expressed as

$$
G R=\frac{N \times I(t)}{d}
$$

\section{Numerical Simulation and Result Analysis}

The numerical simulation assumes the PX event in Maoming, Guangdong, as an example. Maoming is also a place, besides Xiamen, Ningbo, Kunming, and other places, that attracts domestic and foreign attention because of the PX project. The "Maoming PX Incident" has gone through multiple stages of occurrence, development, expansion, and calming down, and it finally subsided when the Maoming local government announced the suspension of the PX project.

According to the statistics of the Social Public Opinion Monitoring and Early Warning System (X-GOT), from March 29 to April 24, 2014, 9558 related news and reports were published (including reprints on news websites) involving the Maoming PX incident in Guangdong, and the main post of the forum was 43,150 . Then, there were 7622 blog posts and 826,844 Weibo main posts (The attention data of each stage is shown in Table 1.) The trend of public opinion on the Internet was significantly different. The peak of public opinion on the Internet occurred on March 31, while the climax of media reports appeared on April 4. According to changes in public opinion trends, the entire public opinion process can be divided into: incubation period 
Table 1 Attention data of each stage of Maoming PX incident

\begin{tabular}{lccccc}
\hline Media Type & Incubation & Explosive & Development & Fallback & Total \\
\hline News & 350 & 3,150 & 5,150 & 908 & 9,558 \\
Forum & 1100 & 21,000 & 17,400 & 3650 & 43,150 \\
Twitter & 302 & 3,600 & 3,200 & 520 & 7,622 \\
Sum & 1752 & 27,750 & 25,750 & 5078 & \\
\hline
\end{tabular}

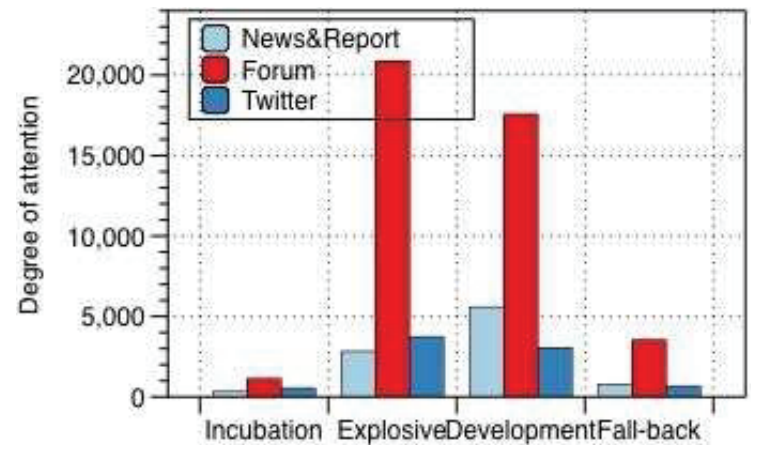

Figure 4 Maoming PX Event Network Public Opinion Attention Trend.

(before March 29), explosive period (March 30-31), development period (April 1-11), and fallback period (April 12 to the present). Related news reports, forums, and microblog public opinionrelated attention are shown in Figure 4.

To verify the accuracy of the model, the data in the aforementioned incidents were used as samples to change the three parameters of incident sensitivity, government recognition, and the Network Public Opinion ecological environment index. The number of contacts was adjusted to find out what happened in emergencies and how should the disposal entity take correct guidance measures to control the spread of Network Public Opinion, so as to provide decision-making ideas for public management decision-makers [29].

As shown in Figure 5, under the combined influence of events, netizens, media, government, and the ecological environment of Network Public Opinion, the popularity of public opinion on public emergencies quickly rises and reaches a peak, and then slowly decreases until it stabilizes at a low level. The changing trend of the spread curve of Network Public Opinion is consistent with the life cycle of public opinion development: during the gestation period of public opinion, only a small number of event-related parties disseminate information, and public opinion is less enthusiastic; subsequently, with the 


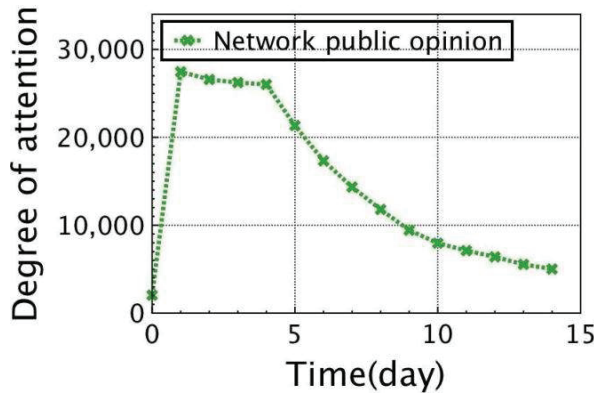

Figure 5 Degree of attention for the Network Public Opinion trend.

spread of event information, a large number of netizens watch and participate. Public opinion quickly enters a period of development and outbreak; finally, the incident is resolved or new problems emerge, and netizens lose their enthusiasm for participating in the incident. With this, public opinion enters a period of decline and disappearance. Under normal circumstances, with the occurrence of new public emergencies or the shift of public attention, public opinion eventually tends to zero.

Now, we firstly adjust any parameter of the following three variables: event sensitivity, government recognition, and Network Public Opinion ecological index. To enhance the comparison of the simulation results, the simulation experiment adjusts these factors to $50 \%$. The sensitivity of the adjustment event can be calculated using Equation (6). In the equation, the contact rate is set to 0.5 and the Network Public Opinion attention is equal to the newly added participants joining the original participants. Amplitude modulation is the ratio of increasing or decreasing sensitivity on the original basis. The data in Table 2 can be obtained through the source data in Table 1 and model calculations.

The impact of adjusting event sensitivity on the popularity of Network Public Opinion is shown in Figure 6. When the event sensitivity is increased by $50 \%$, the resulting simulation curve increases compared with the original public opinion curve; and when the event sensitivity is decreased by $50 \%$, the obtained simulation curve is slightly lower compared with the original public opinion trend curve. It is inferred that event sensitivity is positively correlated with the popularity of Network Public Opinion. Generally speaking, public emergencies with high event sensitivity cause greater damage and destruction to society. Such incidents tend to increase the tension and cohesion of netizens and the media in the process of event dissemination, thereby inspiring netizens to continue to follow and participate in the event with the media. 
Research on Mining and Application of Group Events 1901

Table 2 Dataset distribution

\begin{tabular}{lccc}
\hline Time (Day) & Degree of Attention & Add 50\% & Decrease 50\% \\
\hline 0 & 1,752 & 1,802 & 1,702 \\
1 & 27,750 & 29,138 & 25,363 \\
2 & 26,950 & 28,621 & 24,456 \\
3 & 26,360 & 27,541 & 24,115 \\
4 & 26,019 & 26,887 & 23,108 \\
5 & 21,543 & 23,670 & 18,108 \\
6 & 17,350 & 20,041 & 14,121 \\
7 & 14,132 & 16,101 & 11,512 \\
8 & 12,025 & 14,008 & 9,352 \\
9 & 9,523 & 11,341 & 7,287 \\
10 & 7,912 & 98,41 & 6,225 \\
11 & 7,019 & 7,923 & 5,351 \\
12 & 6,357 & 7,141 & 4,660 \\
13 & 5,650 & 6,489 & 4,321 \\
14 & 5,078 & 6,085 & 4,036 \\
\hline
\end{tabular}

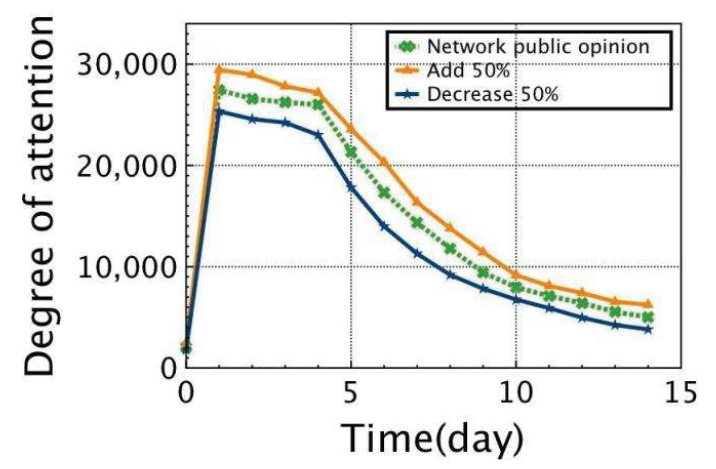

Figure 6 The impact of adjusting event sensitivity.

Once again, let us verify the influence of the number of contacts on the popularity of online public opinion. Assuming that the total number of netizens participating in forums and blog discussions and postings in the PX event is 20,000 people, the contact rate is 20 people per day, the infection ratio is 0.45 and 0.65 , and the average duration of infection spread is 3 days. It is assumed that there is one infection spreader in the initial state, and the others are susceptible to doubt. 


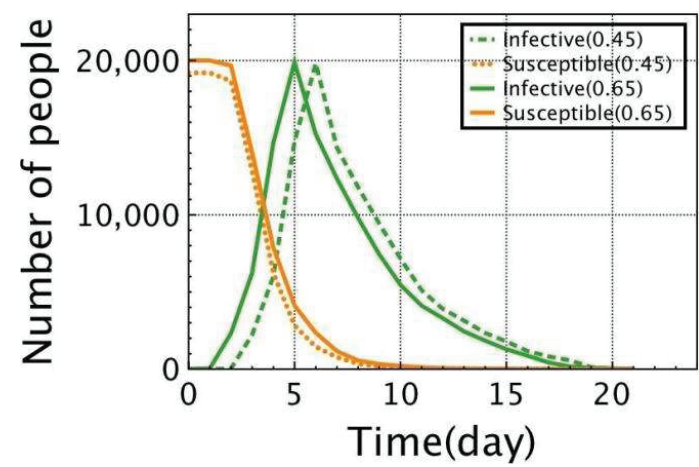

Figure 7 Impact of the number of adjust contacts $\varphi$.

Substituting the parameters into the model, without changing any parameters, the trend of the number of infected, spreading, and susceptible people is shown in Figure 7.

As can be seen from Figure 7, with an increase in the number of exposures, the number of infected and susceptible people is positively correlated with the total number of netizens participating, and the transmission time is correspondingly earlier.

In addition, to demonstrate the advantages of the SIR model through comparative experiments, we introduce the experimental method of the SNO model. The SNO model mainly adjusts the weights of the following three variables: netizens, media, and the government to deduce the changes in online public opinion. To emphasize the comparability, it is necessary to initialize the comparison experiment settings of the conditions of the two methods. Assuming that the total number of netizens participating in forums and blog discussions and postings in the PX incident is 10,000, the contact rate is 20 people per day, the infection ratio is 0.4 , the average duration of the infection spreader is 3 days, and the initial state has 10 infections spreading. However, other people are susceptible to doubts, the government identity accounted for 0.3 , the online public opinion ecological index (equivalent to the media) accounted for 0.3 , while the ratio of the weight of netizens, media, and the government in the SNO model is 0.4:0.3:0.3. When comparing horizontally, the amplitude is adjusted by $10 \%$ on this basis. The experimental results are shown in Figure 8.

Through comparative experiments, the similarity between the SNO and SRI models is that if the government strengthens supervision, early intervention and strengthening supervision will still produce obvious results, and 


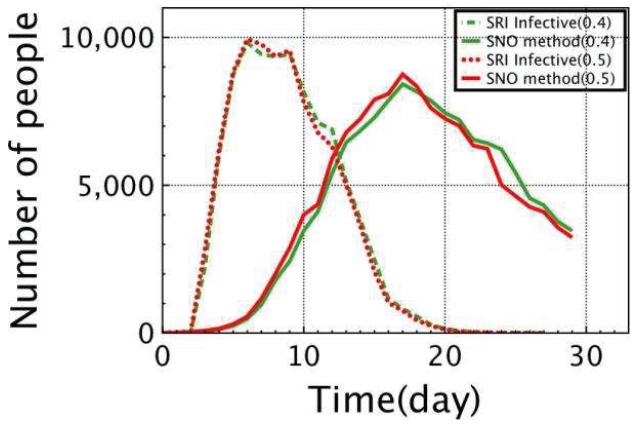

Figure 8 Comparative experiment.

it will affect the attention of Network Public Opinion by sending positive signals to the media and netizens. In the SRI model, when the contact rate is relatively large, the Network Public Opinion quickly reaches its peak. If the government does not take intervention measures, mass incidents occur. On the contrary, in the SNO model, when the government has a large influence weight, the vast majority of netizens and media maintain a consistent attitude with the government, or at least a neutral wait-and-see attitude. Once the influence weights of netizens, media, and the government are relatively close, the attitudes held by netizens and media in the evolution of public opinion fluctuate. Relatively, the convergence of the SRI model is fast.

\section{Conclusion}

We achieved the following: establishment of a system dynamics model based on the SIR spread disease model for the spread of Network Public Opinion, simulation of various public opinions on the network, building of public opinion big data, setting of adjustment parameters through hot data mining settings, and fitting of the simulation results to the actual public opinion event propagation trend better. By simulation the model is validity.

The reduction in contact rate can directly reduce the reduction in the number of infected people spreading and delay the time to reach the peak, reducing the possibility of a Network Public Opinion crisis. Therefore, if necessary, relevant government departments can isolate, delete posts, block, and employ other related methods through the internet. To reduce the rate of contact between netizens, such measures will help reduce the number of people who are infected and spread in extraordinary times. However, if taken for a long time, it may increase public grievances and reduce the credibility 
of the government itself. Therefore, for the government, it is important to fully understand the role of network media and correctly use its advantages to guide the spread of events.

The increase in the rate of government control and guidance, that is, the increase in the rate of cure of infection spreaders, has a positive effect on speeding up the dissipation of public opinion. Therefore, after a mass incident occurs, the government must respond to Network Public Opinion from three aspects: incident sensitivity, increasing government recognition, and optimizing the public opinion environment.

The process of the Network Public Opinion dissemination of mass incidents is relatively complicated, and the model lacks comprehensiveness in revealing the specific influencing factors of the process of dissemination of Network Public Opinion events. The parameter values are set according to the relevant literature and experience, and it is awaiting practical verification and further exploration.

\section{Acknowledgment}

This work is supported by the Natural Science Foundation of Hunan Province, China (2018JJ2084; 2018JJ2371); the Hunan Institute of Technology; the "Three Batches" Top Talent Support Program; the Research Center of Water Resource Management Information; and the Big Data Mining Technology in Chenzhou (yfzx201901); Science and Technology Planning Project in Chenzhou, "Study on the Application of Big Data in Sustainable Use of Water Resources" (zdyf201915).

\section{References}

[1] Du Junfei. Analysis on the Types of Network Group Events. International Press, 2009 (7).

[2] Lu Wengang, Zhang ting. Research on the Management of Online Rumors in Mass Incidents under the Background of Internet - Based on the analysis of the "March 30" PX Event in Maoming, Guangdong. Research on petition and Social Contradictions, 2017, 000(004): 124-142.

[3] Liu Chun-xiang, Jiang Yao-hui. Wise Govern Approach to the Governments Response to Network Mass Disturbances under the Perspective 
of Discourse Theory. Journal of Social Science of Hunan Normal University, 2012(01): 10-13.

[4] Peng bo. Network communitive event rule analysis and guard strategy research. Shanghai: Shanghai jiao tong university, 2008.

[5] Peng Zhihui. Analysis of the Research Path of Internet Public Opinion. Library Journal 000.012 (2016): 63-68.

[6] Shi Bo. Study on the Internal Evolution Mechanism of Public Opinion in Public Crisis Events. Journal of Information 29.004 (2010): 41-45.

[7] Yang Jiuhua. A Discussion on the Mode, Cause and Prevention of Network Mass Incidents. Journal of Chongqing Institute of Socialism 11.4 (2009): 89-92.

[8] He Guoping. The Mobilization Model of Internet Group Incidents and Its Public Opinion Guidance. Research on Ideological and Political Work 09 (2009): 37-39.

[9] Milgram, Travers Stanley. An experimental study of the small world problem. Sociometry 32.4 (1969): 425-443.

[10] Xie yungeng, Liu rui, QIAO Rui, et al. Research Overview of Big Data and Social Public Opinion.New media and society 04 (2014): 134-155.

[11] Fan Guohua, Jia Xiaona. Early-warning mechanism of network Mass disturbance. People's Tribune 000(32) (2010): 158-159.

[12] Liu qiaoling, Li jin, Xiao renbin. Prediction of Internet public opinion spreading trend based on parameter inversion: Taking Sina Weibo as an example. Computer Applications 37(5) (2017): 1419-1423.

[13] Xie shuyun, Quan xiaosong, Shen yuncheng. Construction of the Network Public Opinion evaluation model under the big data environment. Journal of Guiyang University (Natural Science Edition), 2016 (1): $54-57$.

[14] Dong jingwei. Research on influence mechanism in the dynamic evolution process of network opinion based on complex network. Harbin: Harbin Institute of Technology, 2016.

[15] Song huanying. Review on the Application of Big Data in the Network Public Opinion Research in China. Information Search, 2020: 93-99.

[16] Yan daocheng. Research on Network Public Opinion in Mass Incidents. Xinhua Publishing House, 2013.

[17] Yi Xing. Group events and Network Public Opinion Research Review. Journal of Political Science and Law, 2014, 31(4): 101-107.

[18] Lin Ling. Communication Mechanism and Coping Strategies of Network Mass Events. Academia Bimestrie, 2010(5): 19-24. 
[19] Ma yan. Study on the Method of Micro-blogging Public Opinion Hotspots Mining in Big Data. Journal of Modern Information, 2014, 34(11): 29-33.

[20] Jiang xinhao. The guiding strategy of mass incidents network public opinion. Legal system and society 000.007 (2020): 121-122.

[21] Zeng runxi, Wang guohua, Chen qiang. The Governance of Internet Society under the Relationship between State and Society. Journal of Beijing institute of technology Social sciences edition, 2010, 12(5): 121-125.

[22] YuanXue. Innovation of government behavior mode in network social management in the era of big data. Journal of Guangdong Institute of Public Administration, 2013, 25(4): 25-30.

[23] Hou Jundong, Xiao Renbin. Structural Reversal of Network Public Opinion in Collective Behaviors: Internal Mechanism and Actual Representations. Journal of Social Sciences, 2017 (11): 80-90.

[24] Zhang wei, He mingsheng, etc. On the dynamics of network public opinion: A research based on Weisbuch-Deffuant Model. Information Magazine, 2013, pp. 43-48.

[25] Zhang rui. Dilemmas of Cyber Society Governance in China and Corresponding Strategies: From the Perspective of Public Reason. Suzhou: Suzhou university, 2016.

[26] Wang linlin. Research on Dissemination and Government Response of Internet Public Opinion from Unexpected Public Events based on the System Dynamics Model. Shanghai: East China Norma University, 2016.

[27] Zhang mingshan, Zhang yingchun. Effect model of the network public feeling spreading on mass emergencies. Journal of Southwest University for Nationalities Natural Science Edition, 2011, 37(3): 331-335.

[28] Zhai jie. Micro-blog Emergency Network Public Opinion research on Rule discovery and Prediction method. Dalian: Dalian University of Technology, 2016.

[29] Wen hong. Logic Interaction between Public Opinion Orientation and Government Response in Internet Group Crisis Events-Emotional Analysis Based on Big Data of Snow Village Event. Political Studies, 2019 (1): 77-90. 


\section{Biographies}

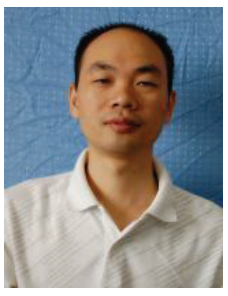

Weimin Gao received the B.E. degrees from Nanhua University, China, in 1999 and the master's degrees from the School of Information Science and Engineering, Hunan University, China,in 2007. He is currently pursuing the Ph.D. degree with the School of Computer Science and Engineering, Central South University, China. His current research interest is data center network.

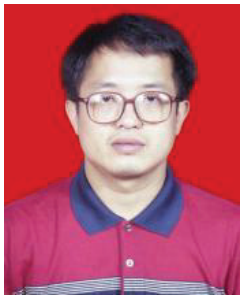

Jiaming Zhong received the B.S. degree from Hunan Normal University. Now he is a professor at Xiangnan University. His research interest is intelligent information processing. He has published more than 40 papers. 
1908 W. Gao et al.

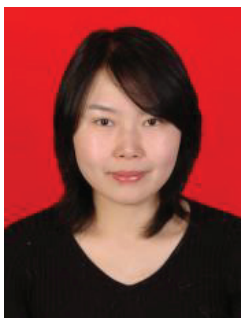

Yuan Xiao received the M.S. degree from Chongqing University of Technology in 2010. Now she is a lecturer at Xiangnan University. Her research interests include Financial Big Data and Intelligent Financial. She has published more than 10 papers. 